\title{
Design and fabrication of segmented, slotted waveguides for electro-optic modulation
}

\author{
Guangxi Wang ${ }^{\text {a) }}$ \\ Department of Electrical Engineering, California Institute of Technology, 1200 E California Blvd., \\ Pasadena, California 91125, USA \\ Tom Baehr-Jones, Michael Hochberg, and Axel Scherer \\ Department of Applied Physics, California Institute of Technology, 1200 E California Blvd., Pasadena, \\ California 91125, USA
}

(Received 20 August 2007; accepted 12 September 2007; published online 1 October 2007)

\begin{abstract}
Recently, slotted waveguides have been used in conjunction with second order nonlinear optical polymers to provide a platform for electro-optic modulation at telecommunication frequencies. A challenge with these devices is the need to provide high-frequency electrical driving signals to the slots. The silicon resistivity must be high to reduce optical loss, and as a result electrical performance can be severely limited. We have designed and fabricated a class of waveguide that achieves quasicontinuous electrical contact with subwavelength segmentations and has low optical losses of $4 \mathrm{~dB} / \mathrm{cm}$, allowing both millimeter-wave and optical fields to be concentrated in the same area. (C) 2007 American Institute of Physics. [DOI: 10.1063/1.2793618]
\end{abstract}

The use of second order nonlinear materials provides an attractive method for achieving modulation at telecommunication wavelengths. As has recently been demonstrated, ${ }^{1}$ the $\chi^{2}$ moment of nonlinear polymers is ultrafast, raising the prospect that extremely high speed modulators can be realized, limited only by the performance of the supporting passive electronics. In fact, practical modulators with speeds on the order of $165 \mathrm{GHz}$ have recently been demonstrated. ${ }^{2}$ In a hybrid slotted waveguide-polymer system, the silicon waveguide acts as a microscale transparent contact, ${ }^{1}$ and the extremely narrow slot provides for a very high electric field per volt. In a recent paper, ${ }^{3}$ we have shown that millivolt-scale optical modulators may now be possible based on such a device geometry. These systems compare favorably to modulators in which the electrodes are stood off from the waveguide and do not touch the optical mode, due to the smaller gap sizes involved and the commensurately larger electric fields per volt. ${ }^{4}$ Even using slots on the order of $100 \mathrm{~nm}$ or more in width, more than five times enhancements in effective index shift have been obtained with slotted waveguides for identical modulation materials and driving voltages, when compared with external-electrode approaches. ${ }^{1,5}$

A major challenge, however, is that for slotted waveguides, the waveguide core must be electrically conductive. If the core is made of silicon, it must be doped to decrease the resistivity if it is to support radio or millimeter-wave propagation over any significant distance. Doping with 2 $\times 10^{18}$ atoms $/ \mathrm{cm}^{3}$ of phosphorus, for example, leads to a resistivity of $0.04 \Omega \mathrm{cm}$, but increases waveguide $\operatorname{loss}^{1}$ by about $10 \mathrm{~dB} / \mathrm{cm}$. For some applications, it might be necessary to dope with an even lower concentration to avoid this loss penalty. To appreciate the limitation that this relatively large resistivity implies, consider the turn-on time of a slotted waveguide based Mach-Zehnder with arm length of $1 \mathrm{~cm}$, edge size of $0.3 \mu \mathrm{m}$, edge height of $0.11 \mu \mathrm{m}$, and a slot size of $0.14 \mu \mathrm{m}$. The rc based turn-on time is approximately $9.5 \mu \mathrm{s}$, leading to a speed limit for the Mach-Zehnder

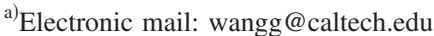

of $17 \mathrm{kHz}$. Clearly, such a slow turn-on time would completely negate the benefit associated with the ultrafast performance of the nonlinear polymers.

Previously, segmented waveguides have been used to provide electrical contacts to nonslotted waveguides. ${ }^{6}$ Marginal waveguide losses were achieved for these structures, in the best case $16 \mathrm{~dB} / \mathrm{cm}$. Such losses were acceptable for the application in question, which required only very short segmented regions. Here, we apply a similar idea to slotted waveguides. We start with a standard slotted waveguide ${ }^{7,8}$ in a $0.11 \mu \mathrm{m}$ thick silicon layer with two $0.3 \mu \mathrm{m}$ sides separated by a $0.14 \mu \mathrm{m}$ slot, as shown in Fig. 1 . The structure is supported by an oxide layer. We note that for regular slotted waveguides on a similar substrate, we have previously obtained waveguide losses ${ }^{9}$ of $10 \mathrm{~dB} / \mathrm{cm}$. This waveguide is also clad with polymethyl methacrylate with an optical index of about 1.5 . $^{10,11}$

We investigated two designs [Table I]. To simulate these designs, we solve Maxwell's equations computationally in the frequency domain. It was found that the propagating mode was confined in the slot region in a fashion very similar to that of a normal slotted waveguide mode. Launching the segmented slotted mode was not difficult because the butt-coupling insertion loss between a normal slotted waveguide and a segmented slotted waveguide was found to be negligible. The nonsegmented mode can be solved with a conventional mode solver. ${ }^{12}$

By observing the propagation of the slotted mode in a $5 \mu \mathrm{m}$ long three dimensional simulation, we were able to determine the dispersion diagram of the slotted mode. This, as well as the solved modal pattern, is shown in Fig. 2. We note that the waveguides are very broadband, having relatively linear dispersion over the course of many tens of nanometers of spectrum. The segmentation leads extend for about $4 \mu \mathrm{m}$ laterally in each direction from the central waveguide, which is sufficient to achieve optical isolation. In a high speed device, we would anticipate constructing a transmission line with metal contacts stood off laterally by at least this distance from the waveguide core. 
(a)
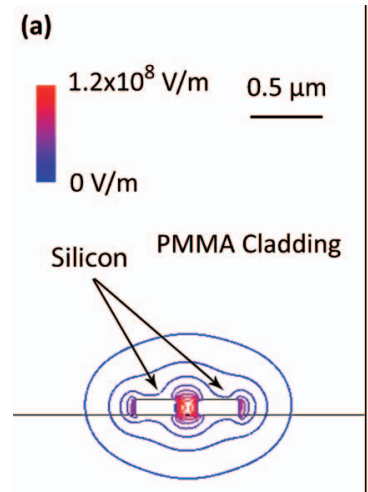

Silicon Dioxide (b)

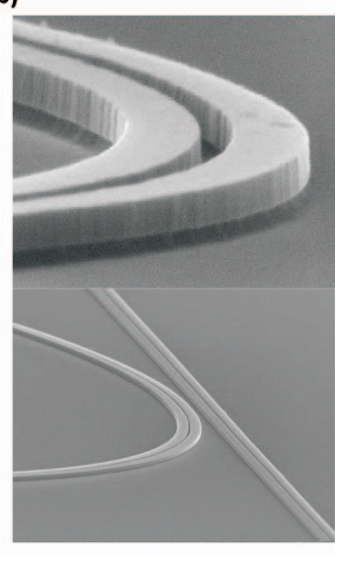

FIG. 1. (Color) (a) shows a diagram of a conventional slotted waveguide, as well as the modal pattern near $1550 \mathrm{~nm}$. The mode is plotted with contours of $10 \%$ in $|E|$, assuming $1 \mathrm{~W}$ of average power flow. (b) shows a scanning electron microscopy (SEM) micrograph of a fabricated slotted waveguide, in this case being used for a ring resonator.

The fabrication procedure for these waveguides is similar to that described in previous publications. ${ }^{6}$ The devices were tested optically using grating couplers and a vertically mounted fiber array. ${ }^{12}$ Devices consisted of a simple loop involving a varying length of segmented slotted waveguides. Approximately 43 devices of each waveguide type were tested. Devices with segmented slotted waveguide lengths from 33 to $8000 \mu \mathrm{m}$ were tested. The values reported in Table I were determined by performing a linear regression on the results. The dominant loss mechanisms in this waveguide system are edge roughness and surface state absorption. ${ }^{13}$ Further development of the lithographic and etching processes, as well as surface treatments, can be expected to decrease the loss even further. ${ }^{13,14}$ Insertion losses from the normal slotted waveguides to the segmented and slotted waveguides were negligibly small.

(a)

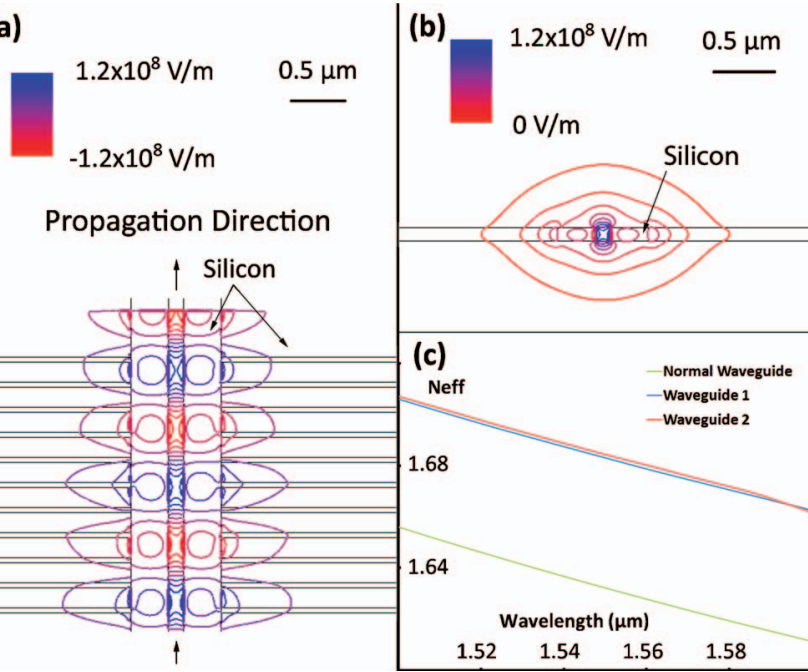

FIG. 2. (Color) (a) shows a vertical cross section of waveguide design 1 for a mode at $1550 \mathrm{~nm}$. The mode is plotted in contours of $10 \%$ in $E_{x}$, assuming $1 \mathrm{~W}$ of average power flow. (b) shows a horizontal cross section of waveguide design 1, with the cross section taken midway through the waveguide. The mode is plotted with contours of $10 \%$ in $|E|$, assuming $1 \mathrm{~W}$ of average power flow. (c) shows the effective index as a function of wavelength for the two segmented slotted waveguide designs, as well as the normal slotted waveguide.

Downloaded 01 Oct 2007 to 131.215.225.176. Redistribution subject to AlP license or copyright, see http://apl.aip.org/apl/copyright.jsp

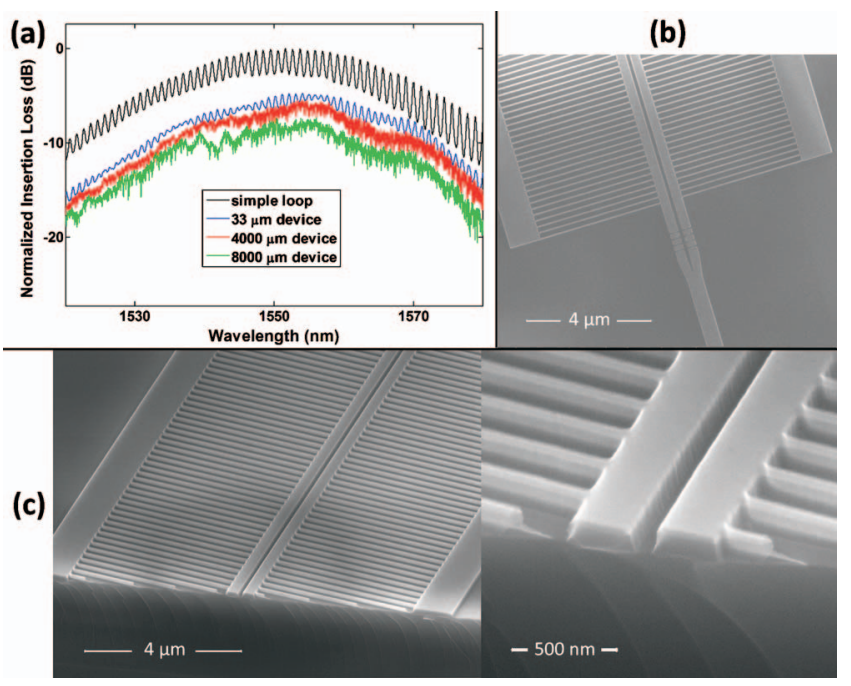

FIG. 3. (Color) (a) shows typical normalized insertion losses of devices in three sizes $(33,4000$, and $8000 \mu \mathrm{m}$ of segmented slotted structures) in $\mathrm{dB}$ vs. wavelength in $\mathrm{nm}$. With the periodicity of design $2,8000 \mu \mathrm{m}$ corresponds to 32000 contact pairs. A nonslotted waveguide control loop device is also presented in the same plot. (b) shows a top view SEM micrograph of a segmented slotted waveguide device. (c) shows two angled view SEM micrographs of the devices exposing their cross-sections.

Figure 3 shows the typical normalized insertion loss for a number of devices. The difference between a simple loop and the segmented slotted devices includes the losses due to the mode conversion from normal ridge waveguides to slotted waveguides, which were measured to be approximately $2 \mathrm{~dB}$ per transition. More efficient ridge to slotted mode converters have recently been designed ${ }^{15}$ which exhibit losses better than $0.25 \mathrm{~dB}$, implying that this loss is not a fundamental limitation to a segmented and slotted waveguide based device. With the grating coupler input curve subtracted, the performance of our waveguides as a function of wavelength is relatively flat. The fine fringes that are visible come from two sources: First, return loss of the grating couplers, and second, the accumulation of loss from weak scatterers, which is visible as narrow loss peaks on the longer devices.

Although it is likely that these waveguides will need to be coupled to transmission lines in practical devices (typical modulator lengths are on the order of millimeters to centimeters), it is possible to make some basic arguments about high-frequency performance for these waveguide geometries. Whatever the geometry of the driving rf transmission line, the unit-cell rc circuit implied in the segmented geometry must be charged. The waveguides we have tested were fabricated with silicon on insulator wafers that were slightly boron doped to $10^{15}$ atoms $/ \mathrm{cm}^{3}$, with resistivity of $20 \Omega \mathrm{cm}$. For the hypothetical resistivity of the doped silicon described at the beginning of this paper, $0.04 \Omega \mathrm{cm}$, we can calculate the charging time to be $2.3 \mathrm{ps}$ for both designs 1 and 2: this

TABLE I. Designs studied in this paper. We also present the measured loss values and speed limits for a $1 \mathrm{~cm}$ long Mach-Zehnder modulator here.

\begin{tabular}{lcccc}
\hline \hline & $\begin{array}{c}\text { Contact } \\
\text { size }(\mathrm{nm})\end{array}$ & $\begin{array}{c}\text { Contact } \\
\text { periodicity }(\mathrm{nm})\end{array}$ & $\begin{array}{c}\text { Measured loss } \\
\text { achieved }(\mathrm{dB} / \mathrm{cm})\end{array}$ & $\begin{array}{c}\text { Intrinsic speed } \\
\text { limit }(\mathrm{GHz})\end{array}$ \\
\hline Design 1 & 40 & 200 & $4.62 \pm 0.11$ & 70 \\
Design 2 & 50 & 250 & $4.00 \pm 0.26$ & 70 \\
\hline \hline
\end{tabular}


corresponds to a speed limit of around $70 \mathrm{GHz}$, as shown in Table I. Though this is only an order-of-magnitude estimate, it provides a strong indication that these designs will be suitable for building devices at $10 \mathrm{GHz}$ and above. In this case, the additional optical losses introduced are anticipated to be comparable to the loss increase seen in doping normal slotted waveguides, ${ }^{1}$ which is about $10 \mathrm{~dB} / \mathrm{cm}$.

We have experimentally demonstrated a low loss waveguide design which allows for the concentration of both optical and $\mathrm{rf} /$ millimeter-wave radiation into the same void nanostructure. We expect these waveguides to find applications in electro-optic polymer based modulators and other nonlinear devices, as well as in the construction of integrated optical sensors.

The authors gratefully acknowledge financial support from DARPA under the MORPH program and the NSF under the Materials and Devices for Information Technology Research center. The authors would also like to acknowledge Cornell Nanoscale Science and Technology Facility.

${ }^{1}$ T. Baehr-Jones, M. Hochberg, G. Wang, R. Lawson, Y. Liao, P. A. Sullivan, L. Dalton, A. K.-Y. Jen, and A. Scherer, Opt. Express 13, 5216 (2005).
${ }^{2}$ B. Bortnik, Y. C. Hung, H. Tazawa, B. J. Seo, J. D. Luo, A. K.-Y. Jen, W. H. Steier, and H. R. Fetterman, IEEE J. Sel. Top. Quantum Electron. 13, 104 (2007).

${ }^{3}$ T. Baehr-Jones, M. Hochberg, G. Wang, J. Huang, P. A. Sullivan, L. Dalton, and A. Scherer, Opt. Express 15, 8401 (2007).

${ }^{4}$ P. Rabiei, W. H. Steier, C. Zhang, and L. Dalton, J. Lightwave Technol. 20, 1968 (2002).

${ }^{5}$ H. Tazawa, Y.-H. Kuo, I. Dunayevskiy, J. Luo, A. K.-Y. Jen, H. R. Fetterman, and W. H. Steier, J. Lightwave Technol. 24, 3514 (2006).

${ }^{6}$ M. Hochberg, T. Baehr-Jones, C. Walker, J. Witzens, L. C. Gunn, and A. Scherer, J. Opt. Soc. Am. B 22, 1493 (2005).

${ }^{7}$ Q. Xu, V. Almeida, R. Panepucci, and M. Lipson, Opt. Lett. 29, 1626 (2004).

${ }^{8}$ Q. Xu, V. Almeida, R. Panepucci, and M. Lipson, Opt. Lett. 29, 1209 (2004).

${ }^{9}$ T. Baehr-Jones, M. Hochberg, C. Walker, and A. Scherer, Appl. Phys. Lett. 86, 081101 (2005).

${ }^{10}$ MicroChem Corp., http://www.microchem.com/products/pmma.htm (2007).

${ }^{11}$ Y.-G. Zhao, W.-K. Lu, Y. Ma, S.-S. Kim, S. T. Ho, and T. J. Marks, Appl. Phys. Lett. 77, 2961 (2000)

${ }^{12}$ T. Baehr-Jones, M. Hochberg, C. Walker, and A. Scherer, Appl. Phys. Lett. 85, 3346 (2004)

${ }^{13}$ M. Borselli, Ph.D. thesis, California Institute of Technology, 2006.

${ }^{14}$ K. K. Lee, D. R. Lim, L. C. Kimerling, J. Shin, and F. Cerrina, Opt. Lett. 26, 1888 (2001)

${ }^{15}$ N.-N. Feng, R. Sun, L. C. Kimerling, and J. Michel, Opt. Lett. 32, 1250 (2007). 